\title{
Evaluation of Bleb Characteristics after Trabeculectomy and Glaucoma Implant Surgery in the Rabbit
}

\author{
Kyoko Ishida $^{a}$ Yukimichi Nakano $^{\mathrm{b}}$ Kazuki Ojino $^{\mathrm{b}}$ Masamitsu Shimazawa $^{\mathrm{b}}$ \\ Tomohiro Otsuka $^{b}$ Satoshi Inagaki ${ }^{b}$ Kazuhide Kawase ${ }^{c}$ Hideaki Hara $^{b}$ \\ Tetsuya Yamamoto ${ }^{c}$ \\ ${ }^{a}$ Department of Ophthalmology, Toho University Ohashi Medical Center, Tokyo, Japan; \\ ${ }^{\mathrm{b}}$ Molecular Pharmacology, Department of Biofunctional Evaluation, Gifu Pharmaceutical University, Gifu, Japan; \\ 'Department of Ophthalmology, Gifu University Graduate School of Medicine, Gifu, Japan
}

\section{Keywords}

Glaucoma · Glaucoma surgery · Bleb · Inflammation

\section{Abstract}

The characteristics of the conjunctival bleb are some of the most important factors for the surgical success of glaucoma filtering surgery. To improve surgical outcome, we investigated bleb histology after 3 different glaucoma surgeries. Surgery was performed in 21 white rabbits. Rabbits were randomized to trabeculectomy or implantation with EX-PRESS or a silicone tube (each $n=7$ ). Bleb survival, intraocular pressure (IOP), and vascularity were evaluated. At 6 weeks, eyes were enucleated for histological analysis. Postoperative IOP at 2 weeks was significantly lower in the trabeculectomy and the EX-PRESS group than in the silicone tube group ( $p=$ 0.037 ) but not thereafter. Postoperative bleb survival ( $p=$ $0.542)$ and vascularity $(p=0.988)$ were similar among the 3 groups. Histologically, a capsule showing mild fibroblast proliferation associated with intercellular collagen was present around the surgical site. The thickness of the bleb was similar among all experimental groups, but it was signifi-

karger@karger.com www.karger.com/ore

Karger $\stackrel{\text { ' }}{5}$

BOPEN ACCESS
(C) 2020 The Author(s)

Published by S. Karger AG, Basel

This article is licensed under the Creative Commons AttributionNonCommercial-NoDerivatives 4.0 International License (CC BYNC-ND) (http://www.karger.com/Services/OpenAccessLicense) Usage and distribution for commercial purposes as well as any distribution of modified material requires written permission. cantly greater than in controls $(p<0.05)$. The inflammatory area did not differ between the EX-PRESS and the silicone tube group but was significantly greater in the trabeculectomy group than in the tube group $(p=0.031)$. A correlation between the thickness of the bleb wall and inflammation was found ( $r=0.56, p<0.01)$. EX-PRESS and silicone tube implants appear to be relatively inert, with little difference in biocompatibility and bleb survival. Since some degree of inflammation was still observed histologically in the bleb, more noninvasive surgical methods and more biocompatible materials may be desirable.

(C) 2020 The Author(s)

Published by S. Karger AG, Basel

\section{Introduction}

When maximal tolerable medical therapy and/or laser surgery fail to lower intraocular pressure (IOP) sufficiently to prevent optic nerve damage or visual field deterioration, glaucoma filtration surgery is performed to reduce IOP [1]. Trabeculectomy, in which a fistula is created to allow aqueous humor to drain into a conjunctival 
filtering bleb, is the most commonly performed glaucoma filtration surgery [2]. However, $30-50 \%$ of surgical procedures fail due to postoperative fibrosis-related fistula block and bleb failure [3].

Glaucoma drainage devices (GDDs) have been introduced in an effort to improve fluid outflow and offer an alternative to traditional filtration surgery [4]. The use of GDDs, such as the EX-PRESS glaucoma filtration device (Alcon Laboratories, Fort Worth, TX, USA), the Baerveldt glaucoma implant (Abbott Medical Optics, Abbott Park, IL, USA), and Ahmed glaucoma valve (New World Medical, Rancho Cucamonga, CA, USA), has increased recently.

The EX-PRESS device is a nonvalved, stainless-steel device that lowers IOP by shunting aqueous humor from the anterior chamber into the subconjunctival space adjacent to the limbus. The surgical technique is similar to standard trabeculectomy, and involves the creation of a scleral flap and a conjunctival filtration bleb, but neither sclerectomy nor iridectomy is required when implanting the device. Published clinical studies [5-8] have shown that use of EX-PRESS and trabeculectomy have comparable efficacy in IOP reduction, medication reduction, and qualified surgical success rate. However, EX-PRESS has been shown to have lower rates of postoperative complications associated with hypotony and bleeding than trabeculectomy. Despite the evidence for the clinical utility of this device, its biocompatibility, local fibrosis reaction, and bleb morphology, associated with the presence of a stainless-steel device, elimination of trabeculectomy and iridectomy, and more precise and controlled outflow due to the unified 50- $\mu$ m lumen of the device, are still not fully understood $[4,9-13]$. To date, only 2 studies and 1 case report [11-13] have evaluated bleb morphology with the current EX-PRESS model: P-50.

The Baerveldt glaucoma implant and the Ahmed glaucoma valve are other GDDs that consist of a silicone tube and a plate, and aqueous humor is drained from the anterior chamber through the tube to the subconjunctival space around the plate located at the equatorial sclera. Both devices are used after trabeculectomy failure or in complicated glaucoma cases, in which the rate of failure of simple filtration surgery is high. However, the most common reason for surgical failure of the Baerveldt implant and Ahmed valve is the formation of a thick fibrous capsule around the device, which precludes aqueous absorption from the conjunctiva $[14,15]$.

The characteristics of the conjunctival bleb are some of the most important factors for the surgical success of glaucoma drainage surgery. Eye inflammation and scar- ring, which are due in part to implant bioincompatibility [16-19] and the degree of trauma sustained by the eye during implantation, can be assessed by histological and clinical parameters. To improve surgical results of filtering surgery, in this study, we focused on histopathological evaluation of rabbit eyes after conventional trabeculectomy, EX-PRESS P-50 implantation, and a silicone tube implant, and compared inflammatory responses following these 3 procedures in the subconjunctival space of the rabbit, as no study to date has compared differences in the histological and clinical parameters among the 3 types of filtering surgeries in an experimental model at the same time.

\section{Materials and Methods}

\section{Study Design}

The study used 13-week-old male white rabbits $(2.0-3.0 \mathrm{~kg}$; SLC, Shizuoka, Japan). The rabbits were housed under controlled lighting conditions (12-h light:12-h dark). Twenty-one rabbits were divided into 3 groups: a group that underwent trabeculectomy $(n=7)$, a group that underwent filtration surgery with EXPRESS P-50 device implantation $(n=7)$, and a group that underwent filtration surgery with a Baerveldt glaucoma tube implantation $(n=7)$. All surgeries were performed on the right eye of each rabbit by a single surgeon (K.I). The left eyes of the rabbits served as control for the respective groups.

\section{Trabeculectomy Technique}

The animals were anesthetized with an intramuscular injection of ketamine hydrochloride (Ketalar; $40 \mathrm{mg} / \mathrm{kg}$ body weight; Daiichi Sankyo, Tokyo, Japan) and xylazine hydrochloride (Celactal; $20 \mathrm{mg} / \mathrm{kg}$ body weight; Bayer Health Care, Tokyo, Japan) as well as topical anesthesia (2\% lidocaine gel) before initiation of surgery. The right eye was washed with topical povidone, and a lid speculum was placed to hold the lids open. Then, a 7-mm conjunctival incision was made along the superior corneal limbus with spring microscissors to create a fornix-based conjunctival flap. A partialthickness, trapezoidal-base, 3-mm scleral flap was created in the superior sclera, hinged at the limbus, and the scleral flap was then dissected forward to the limbus. A sponge soaked with mitomycin C (MMC; 0.4 mg/mL; Kyowa Hakko Kirin, Tokyo, Japan) was applied to the scleral flap and subconjunctival tissue for $5 \mathrm{~min}$. Then, MMC was removed by flushing with saline $(20 \mathrm{~mL}$; Otsuka, Tokyo, Japan). An ophthalmic knife was inserted into the anterior chamber from underneath the scleral flap, creating an ostomy, and the entrance was enlarged using a Kelly Descemet membrane punch (Inami, Tokyo, Japan). Forceps were used to pull the peripheral iris through the ostomy site, and a peripheral iridectomy was created using scissors. Two sutures, using 10-0 nylon (MANI, Utsunomiya, Japan), were placed in the corner of the scleral flap. Then, the conjunctival flap was closed using 10-0 nylon sutures. After completion of surgery, ofloxacin ointment (Tarivid; Santen, Osaka, Japan) was applied to the wound and no additional medication was given postoperatively. 
EX-PRESS Technique

EX-PRESS implant surgery was performed as described above for trabeculectomy up to the point of flushing out MMC. Then, a 25 -gauge needle was inserted into the anterior chamber from underneath the scleral flap. A model P-50 EX-PRESS device was inserted into the anterior chamber through the 25 -gauge needle incision. The scleral flap and conjunctival flap were sutured using 10-0 nylon by the same manner done in trabeculectomy. After completion of the surgery, ofloxacin ointment was also applied, and no additional medication was given postoperatively.

\section{Tube Implant Surgery}

Tube implant surgery was the same as described above for trabeculectomy up to the point of scleral flap dissection. MMC was not applied. A 23-gauge needle was then inserted into the anterior chamber from underneath the scleral flap. A silicone tube cut from the Baerveldt implant was inserted into the anterior chamber through the 23-gauge needle incision. The opposite side of the tube was secured to the scleral bed with one 10-0 nylon suture, and the scleral flap was sutured with two 10-0 nylon sutures. A 2-mm-long tube tip was placed under the conjunctival flap without covering by the scleral flap, and fluid efflux into the subconjunctival space was confirmed. The conjunctival flap was sutured using 10-0 nylon, and ofloxacin ointment was also applied at the end of the surgery. Lipcoard or tube ligation was not used in cases with tube surgery. No additional medication was given postoperatively.

\section{IOP Measurements}

Rabbits were anesthetized with topical $0.4 \%$ oxybuprocaine hydrochloride (Benoxil ophthalmic solution 0.4\%; Santen Pharmaceutical Co., Ltd, Osaka, Japan). After confirming sufficient anesthesia, IOP was measured using the TONOVET ${ }^{\mathrm{TM}}$ rebound tonometer (I-care Finland Oy, Vantaa, Finland) prior to surgery and again every week after surgery for 6 weeks. IOP was measured 3 times at each examination and averaged for analysis.

\section{Bleb Evaluation}

Conjunctiva and bleb photographs were taken with a digital camera (Pentax WG-3; Richo, Tokyo, Japan), and handheld slit lamp examinations were conducted to evaluate bleb height before surgery and every week after surgery. The length of the tube was $5 \mathrm{~mm}$ from the limbus; conjunctiva and blebs were evaluated between the limbus to $8 \mathrm{~mm}$ from the limbus. Bleb area and vascularity were classified using the Moorfields Bleb Grading System.

\section{Histology}

All animals were euthanized at the end of the 6-week study. All eyes were then removed. To quantify the amount of collagen and cytoplasm, the eyeballs were embedded in paraffin, and 2 paraffinembedded sections (thickness: $5 \mu \mathrm{m}$ ) were taken through the surgery region of each eye and in the corresponding area of control eyes, and stained with Masson's trichrome (Sigma-Aldrich Co., St. Louis, MO, USA), in which blue staining represented the collagen and red staining the cytoplasm. The sections were then photographed using a microscope (BX50; Olympus, Tokyo, Japan) with a cooled charge-coupled device camera (DP30BP; Olympus; $1,360 \times 1,024$ pixels), and compared morphometrically using MetaMorph $^{\mathrm{TM}}$ software, V7.5.5.0. The thickness of collagen in the sclera was measured at a distance of 0,500 , and $1,000 \mu \mathrm{m}$ from the connection between the corpus ciliare and the sclera. The thickness of the bleb was taken as the mean of the 3 highest bleb measurements from the sclera along the bleb wall [20]. The area of inflammation was measured at the mass of infiltrated cytoplasm in the stained section. Data were averaged from each eye, and the thickness of the collagen in the sclera, the thickness of the bleb, and the inflammatory area were compared among groups.

\section{Statistical Analysis}

Data are presented as means \pm standard error of the mean (SE). IOP, bleb vascularity, and bleb survival time were compared using the Kruskal-Wallis/Mann-Whitney U test with Bonferroni post hoc test among 3 and between 2 groups, respectively. Postoperative IOP was analyzed using Dunnett's test, using the preoperative/ no-surgery values as the respective controls. Statistical comparisons for histological examinations were performed using Student's $t$ test. JSTAT software (Vector, Tokyo, Japan) was used for statisti$\mathrm{cal}$ analysis. Values of $p<0.05$ were considered statistically significant.

\section{Results}

There was no occurrence of endophthalmitis, extrusion of implants, corneal epithelial defects, persistent anterior chamber inflammation, or untimely death of the animals.

\section{Pre- and Postoperative IOP}

The time course of IOP is shown in Figure 1. The preoperative IOP in the trabeculectomy, EX-PRESS, and tube groups was $11.14 \pm 1.38,10.85 \pm 1.77$, and $10.57 \pm$ $0.92 \mathrm{~mm} \mathrm{Hg}$ (means $\pm \mathrm{SE}$ ), respectively. The postoperative IOP in the trabeculectomy group at $1,2,3,4,5$, and 6 weeks was $6.57 \pm 0.48,4.71 \pm 0.87,5.14 \pm 0.83,5.29 \pm$ $0.75,5.86 \pm 0.80$, and $6.43 \pm 0.57 \mathrm{~mm} \mathrm{Hg}$, respectively. The postoperative IOP at these respective times in the EX-PRESS group was $6.43 \pm 0.78,6.43 \pm 1.00,6.57 \pm 1.31$, $7.00 \pm 0.79,7.43 \pm 0.81$, and $8.00 \pm 0.49 \mathrm{~mm} \mathrm{Hg}$. In the tube group, the respective postoperative IOP values were $8.43 \pm 0.95,8.43 \pm 0.84,6.72 \pm 0.87,6.43 \pm 0.84,7.00 \pm$ 0.95 , and $7.71 \pm 0.57 \mathrm{~mm} \mathrm{Hg}$. In the trabeculectomy group, the IOP decreased significantly after surgery (all $p<0.01)$. In the EX-PRESS group, the IOP decreased significantly after surgery, except for the 6-week IOP. In the tube group, the IOP decreased significantly after surgery, except at 1 and 2 weeks.

Among the 3 surgical groups, IOPs were not significantly different, except for IOPs 2 weeks after surgery $(p=0.033)$. More specifically, a significant difference was observed only between the trabeculectomy and the tube group 2 weeks after surgery $(p=0.011)$. 


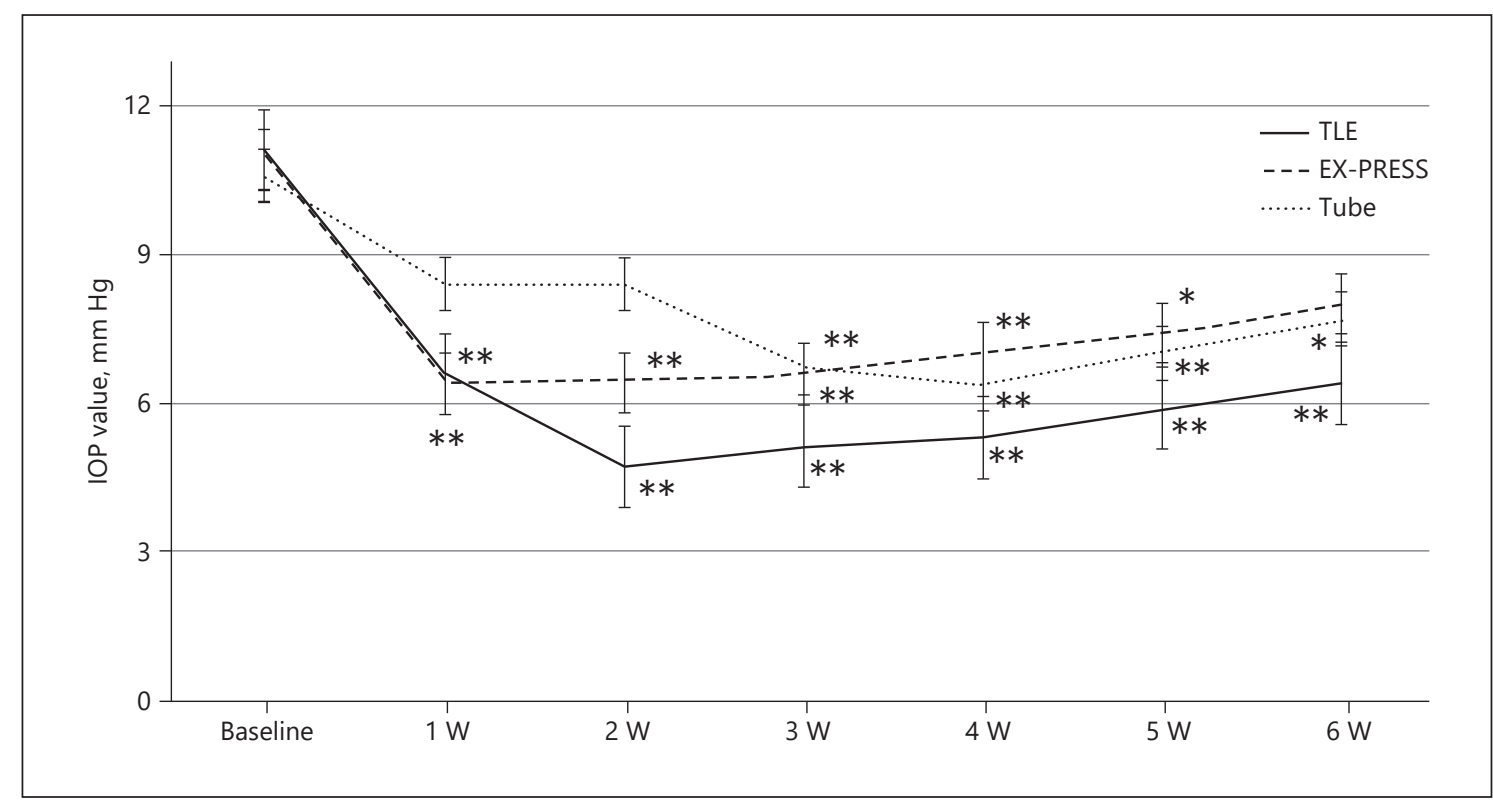

Fig. 1. Time course of intraocular pressure (IOP) among all experimental groups. Solid line, trabeculectomy group; broken line, EX-PRESS group; dotted line, silicone tube group. Means \pm SE. $n=7,{ }^{*} p<0.05,{ }^{* *} p<0.01$, vs. nonoperated eyes, Dunnett's test.

Table 1. Comparison of bleb vascularity among all experimental groups

\begin{tabular}{lllll}
\hline $\begin{array}{l}\text { Trabeculectomy } \\
(n=7)\end{array}$ & $\begin{array}{l}\text { EX-PRESS } \\
(n=7)\end{array}$ & $\begin{array}{l}\text { Silicone tube } \\
(n=7)\end{array}$ & $p$ value \\
\hline $3 \mathrm{~W}$ & $0.29 \pm 0.19$ & $0.14 \pm 0.14$ & $0.71 \pm 0.29$ & 0.064 \\
$6 \mathrm{~W}$ & $1.00 \pm 0.22$ & $1.29 \pm 0.36$ & $1.43 \pm 0.20$ & 0.447 \\
\hline
\end{tabular}

Means \pm SE. Bleb vascularity was judged at 3 and 6 weeks (W) using the grading score of central bleb vascularity of the Moorfields Bleb Grading System. 0, avascularity ( $>50 \%$ avascularity); 1 , reduced vascularity ( $<50 \%$ avascularity); 3 , normal vascularity; 4 , increased vascularity ( $<50 \%$ increased vascularity); 5 , hypervascularity ( $>50 \%$ increased vascularity); $n$, number.

Table 2. Comparison of postoperative bleb survival among all experimental groups

\begin{tabular}{llll}
\hline $\begin{array}{l}\text { Trabeculectomy } \\
(n=7)\end{array}$ & $\begin{array}{l}\text { EX-PRESS } \\
(n=7)\end{array}$ & $\begin{array}{l}\text { Silicone tube } \\
(n=7)\end{array}$ & $p$ value \\
\hline $5.71 \pm 0.29$ & $5.71 \pm 0.29$ & $5.43 \pm 0.30$ & 0.542 \\
\hline
\end{tabular}

Means \pm SE (weeks). $n$, numbers.

\section{Analysis of Filtering Blebs}

Bleb vascularity was increased at 3 weeks in the tube group versus the other groups, but this difference was not statistically significant. Bleb vascularity was similar among all experimental groups at the 3- and 6-week evaluation time points (Table 1). Postoperative bleb survival was also similar among all experimental groups at the 6 -week evaluation time points (Table 2). The average bleb survival in the trabeculectomy group was $5.71 \pm 0.29$ weeks. The EX-PRESS and the tube groups had an average bleb survival of $5.43 \pm 0.20$ and $5.43 \pm 0.30$ weeks, respectively. These differences were not statistically significant $(p=0.542)$.

\section{Histological Analysis of Bleb Filtration}

First, we performed Masson's trichrome staining to elucidate the quantity of collagen deposition by assessing staining density (blue). Moreover, we evaluated inflammatory cytoplasm infiltration (red), which indicated the grade of inflammation throughout the blebs in all groups. In the normal control eyes, the conjunctiva exhibited weak blue staining, and the sclera displayed a little densely packed collagen with a small amount of intense blue staining. In all experimental groups, the subepithelial connective tissue of the conjunctiva showed strong blue staining, and the sclera displayed densely packed collagen 


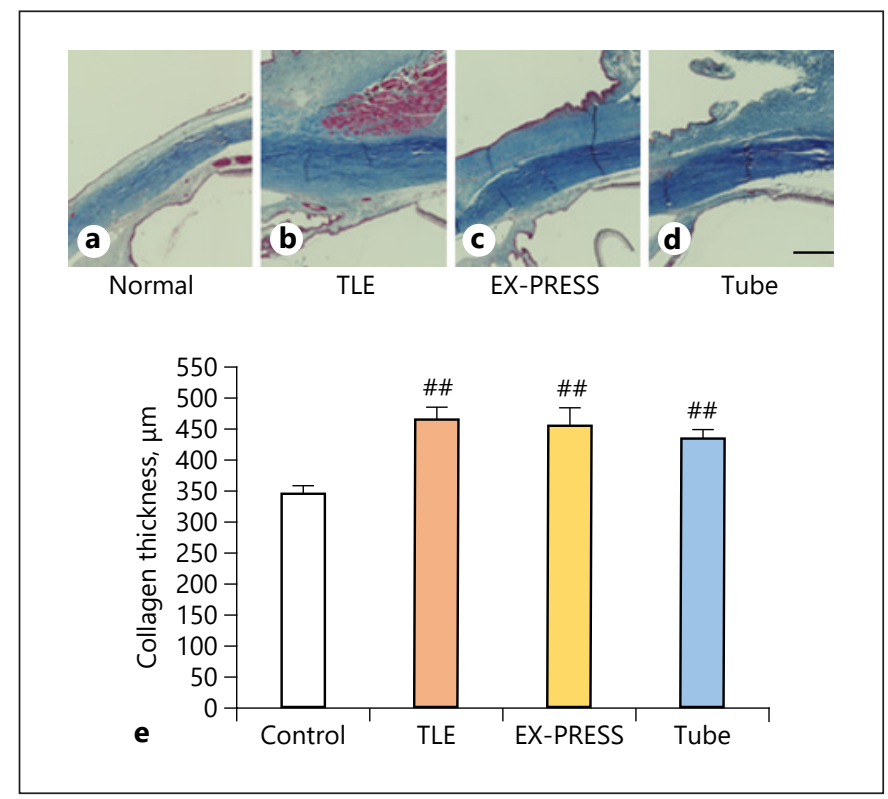

Fig. 2. Comparison of postoperative collagen thickness in sclera among all groups. Representative pictures show Masson's trichrome staining of the sclera. Blue indicates collagen. a Control group. b Trabeculectomy group (TLE). c EX-PRESS group. d Silicone tube (Tube) group. e Collagen thickness in the sclera was significantly increased in all experimental groups compared to the control group. However, collagen thickness did not differ among the experimental groups. Means \pm SE ( $n=7$ in each experimental group and 21 in the control group). ${ }^{\# \#} p<0.01$ vs. control group (Student $t$ test). Bar, $500 \mu \mathrm{m}$.

with intense blue staining. The thickness of collagen in the sclera was significantly increased in all experimental groups $(p<0.01)$. However, collagen thickness did not differ among the experimental groups (Fig. 2). The average collagen thickness was $343.7 \pm 13.4 \mu \mathrm{m}$ in the control eyes; in eyes that underwent trabeculectomy, it was $458.3 \pm 23.6 \mu \mathrm{m}$; in EX-PRESS-implanted eyes, it was $451.5 \pm 26.9 \mu \mathrm{m}$; and in eyes that were implanted with the silicone tube, it was $434.5 \pm 13.2 \mu \mathrm{m}$.

Furthermore, the thickness of the bleb was similar among all experimental groups, but it was significantly increased in all experimental eyes versus control eyes $(p<0.05$; Fig. 3). The average thickness of the bleb (i.e., the conjunctival thickness) in control eyes was $621.2 \pm$ $97.1 \mu \mathrm{m}$. The average thickness of the bleb in eyes that underwent trabeculectomy was 2,034.0 $\pm 196.3 \mu \mathrm{m}$. In EX-PRESS- and the silicone tube-implanted eyes, the average thickness of the bleb was 1,682.0 \pm 171.6 and $1,401.4 \pm 279.6 \mu \mathrm{m}$, respectively.

The area of inflammation in all experimental groups was significantly increased versus the control group.
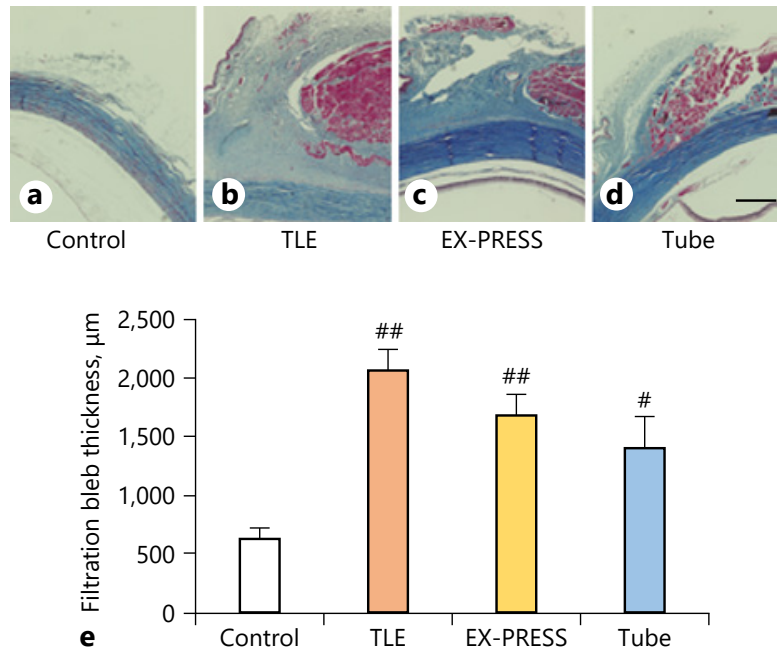

Fig. 3. Comparison of postoperative thickness of filtration blebs among all groups. Representative pictures show Masson's trichrome staining of filtration blebs. Blue indicates collagen and red cytoplasm. a Control group. b Trabeculectomy group (TLE). c EX-PRESS group. d Silicone tube (Tube) group. e Thickness of the filtration bleb was significantly increased in all experimental groups compared to the control group but did not differ among the experimental groups. Means $\pm \mathrm{SE}(n=7$ in each experimental group and 21 in the control group). ${ }^{\# \#} p<0.01,{ }^{\#} p<0.05$, vs. control (Student $t$ test). Bar, $500 \mu \mathrm{m}$.

However, the inflammatory area was not significantly different between the groups implanted with EX-PRESS and silicone tube. The inflammatory area in the trabeculectomy group was significantly increased versus the tube group ( $p=0.031$; Fig. 4 ). The average inflammatory area in the control group was $3,239.5 \pm 3,239.5 \mu \mathrm{m}^{2}$ and $30,42,248.9 \pm 6,98,762.4 \mu^{2}$ in the trabeculectomy group. Eyes implanted with the EX-PRESS device and the tube possessed an average inflammation cell area of $22,48,055.6 \pm 5,64,026.3$ and $11,03,354.5 \pm 3,75,216.5$ $\mu \mathrm{m}^{2}$, respectively. Furthermore, we found that there was a correlation between bleb wall thickness and inflammation ( $r=0.56, p=0.009$; Fig. 5).

\section{Discussion}

Conjunctival bleb failure secondary to scar formation and fibrosis is a common cause of surgical failure after both trabeculectomy and GDDs. The histocompatibility of different biomaterials has been investigated in previous 


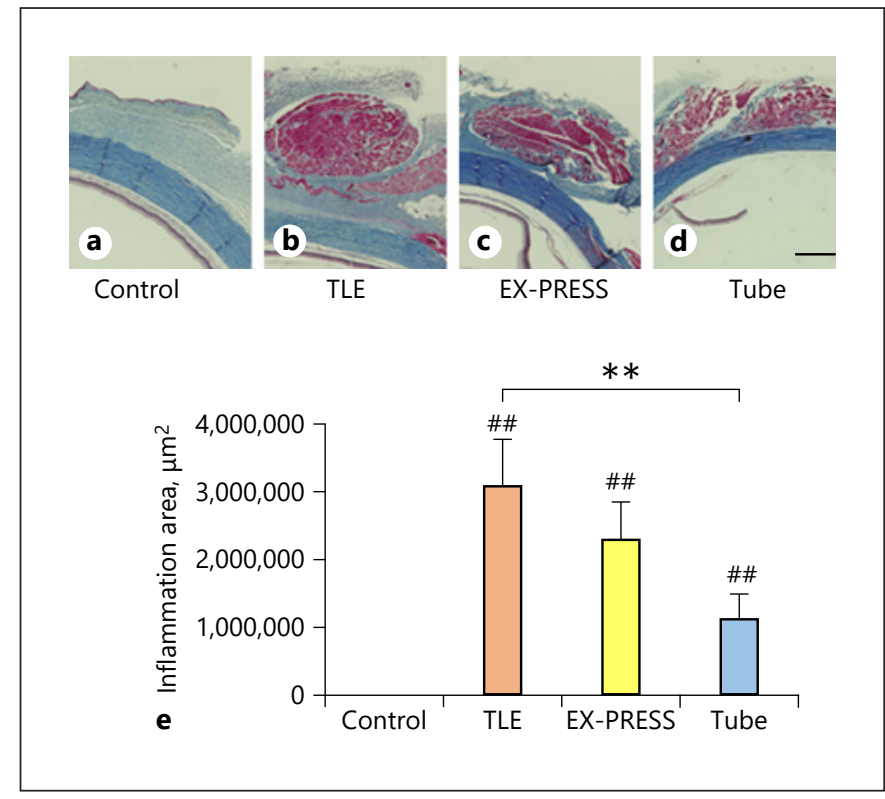

Fig. 4. Comparison of postoperative inflammatory area thickness among groups. Representative pictures show Masson's trichrome staining of the inflammation area. Blue indicates collagen and red indicates cytoplasm. a Control group. b Trabeculectomy group (TLE). c EX-PRESS group. d Silicone tube (Tube) group. e The inflammatory areas in all experimental groups were significantly increased versus the control group. Moreover, it was significantly bigger in the trabeculectomy than the silicone tube group. Means $\pm \mathrm{SE}$ ( $n=7$ in each experimental group). ${ }^{\# \#} p<0.01$ vs. control group, ${ }^{* *} p<0.05$ vs. TLE group (Student $t$ test). Bar, $500 \mu \mathrm{m}$.

studies $[4,9-11,13,16-18]$, and variable degrees of inflammation and fibrosis have been demonstrated. An ideal GDD would consist of an inert biomaterial with similar or better IOP control capability as standard trabeculectomy. In this study, we compared IOP, bleb morphology, and histology in samples of 3 surgical groups: standard trabeculectomy with MMC, EX-PRESS P-50 implant surgery with MMC, and a silicone drainage tube implant (commonly Baerveldt, Ahmed, and Molteno implants).

IOP decreased significantly after surgery in all 3 experimental groups. Postoperative IOP at 2 weeks was significantly lower in the trabeculectomy group than in the tube group; however, this difference was not observed thereafter. Shobayashi et al. [13] evaluated postoperative changes in blebs and levels of aqueous MCP-1 (monocyte chemotactic protein-1) after trabeculectomy versus EX-PRESS P-50 shunt surgery in 6 rabbit eyes. They concluded that there was no significant IOP difference between the 2 groups at any time point. Direct comparison between our study and other studies is difficult; however, we also showed that IOP reduction efficacy was similar among the 3 groups.
In the current study, postoperative bleb survival and average bleb vascularity were similar among the 3 groups. Laing et al. [4] evaluated bleb characteristics in white rabbits after EX-PRESS implantation (model type unidentified) with MMC and silicone tube implantation without an untreated control. Bleb vascularity, evaluated using the Moorfields Bleb Grading System, was similar between the 2 groups $(1.55 \pm 1.03$ in EX-PRESS vs. $1.43 \pm 1.72$ in the tube group) at 6 weeks. Using the same grading system, bleb vascularity was similar in the current study and the study by Laing et al. [4] at 6 weeks $(1.00 \pm 0.22$ in the trabeculectomy, $1.29 \pm 0.36$ in the EX-PRESS, and $1.43 \pm 0.20$ in the tube group). Postoperative bleb survival was $32.15 \pm$ 4.32 days in the EX-PRESS group and $34.30 \pm 2.21$ days in the tube group in the study by Laing et al. [4]. In the current study, postoperative bleb survival was $39.6 \pm 5.6$ in the trabeculectomy group, $37.8 \pm 3.5$ in the EX-PRESS group, and $37.8 \pm 4.0$ in the tube group. In another study [13], the bleb was evaluated using both the Moorfields Bleb Grading System and AS-OCT (anterior-segment optical coherence tomography). There were no significant differences in the inflammatory area or vascularity between the trabeculectomy and EX-PRESS groups at any time point after surgery according to the grading system, which is similar to our result. They, however, noted that the intensity of the bleb wall was significantly lower 1 week after surgery in the EX-PRESS group than in the trabeculectomy group based on AS-OCT evaluations. A retrospective study in humans reported that bleb height after EX-PRESS P-50 implantation was lower in the first 3 months postoperatively, and bleb shape was more diffuse by 3-18 months than after trabeculectomy based on the Moorfields Bleb Grading System [21]; however, there was no significant difference in IOP between the 2 groups throughout the study period. Because bleb structures may reflect future IOP control, further investigations are required.

To study biocompatibility, Nyska et al. [9] implanted an old model of the EX-PRESS device through full-thickness sclera into the anterior chamber without creation of a scleral flap, application of MMC, or conjunctival closure with sutures in 8 rabbit eyes, and they measured the thickness of the fibrotic capsule surrounding the device. The thicknesses of the fibrotic capsule were between 100 and $400 \mu \mathrm{m}$ at 3 months and between 100 and $800 \mu \mathrm{m} 6$ months after EX-PRESS implantation. The authors concluded that EX-PRESS implantation resulted in minimal capsular reaction. Aqueous humor contains various cytokines and chemical mediators that significantly influence the local cellular response [20,23]. Additionally, aqueous humor may play a role in wound healing in vivo [23]. Di- 
Fig. 5. Bleb wall thickness and inflammation area are correlated (Pearson correlation analysis): $r=0.56, p=0.009$.

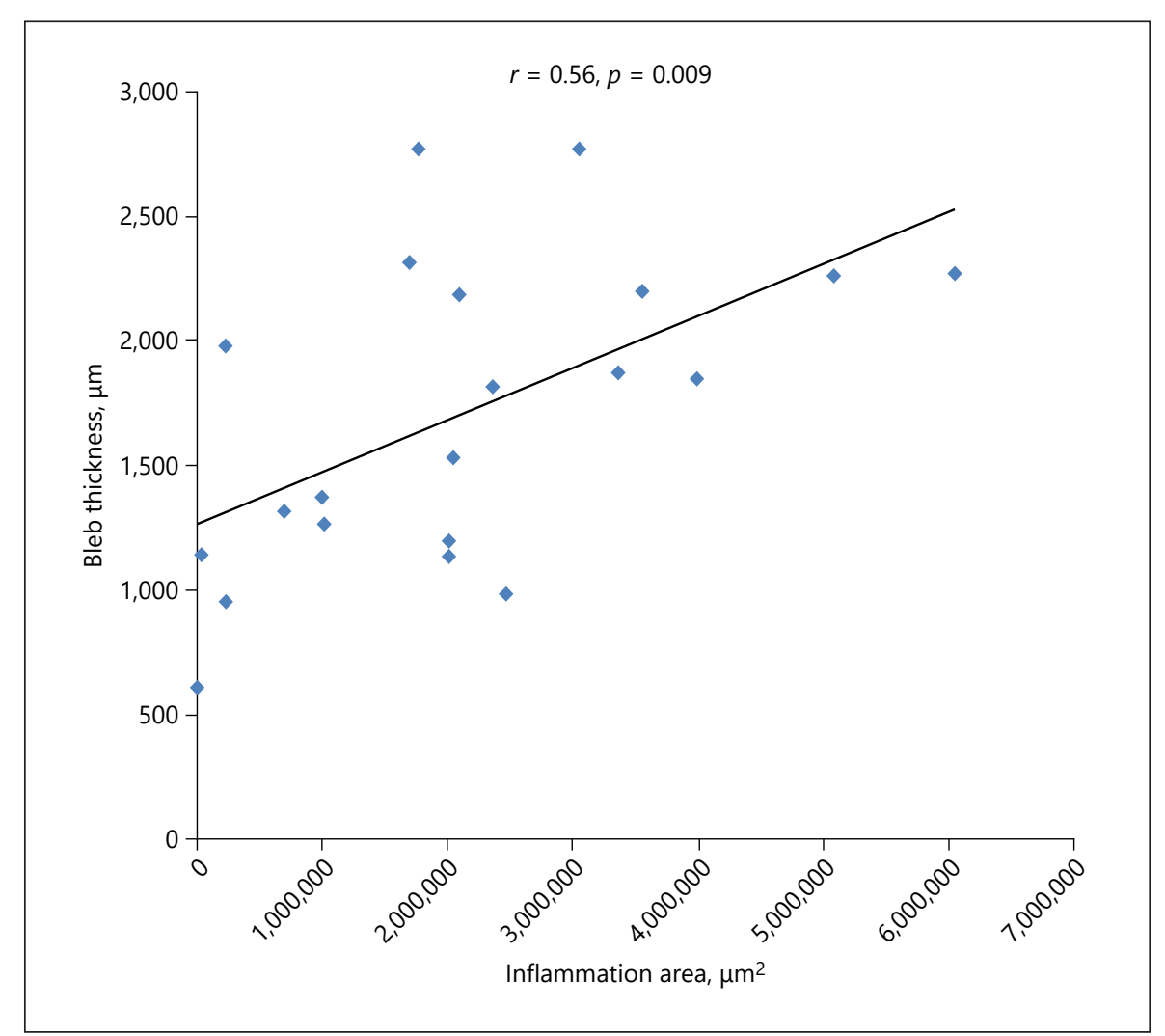

lute aqueous humor stimulates proliferation of cultured fibroblasts [6]. Thus, conjunctival thickness and the characteristics of the bleb capsule after filtering surgery is affected by the aqueous humor, because those devices are designed to allow the flow of aqueous humor into the subconjunctival space.

In our study, we performed 3 surgical procedures to create a filtering bleb, and the thickness of the bleb wall was measured. Although the subepithelial connective tissue and bleb wall thickness were thicker in the 3 experimental groups than in the control eyes, the average thicknesses did not significantly differ among the experimental groups. In all experimental groups, inflammatory areas were significantly increased versus normal eyes. However, inflammatory areas did not differ between the EX-PRESS and the tube group, and it was smaller in the tube than in the trabeculectomy group $(p=0.031)$. This indicates that although EX-PRESS and a silicone tube are foreign bodies, both implants appear to be relatively inert in the aqueous humor environment. However, we found that there was a correlation between bleb wall thickness and inflammation in all cases. Compared to controls, bleb inflammation induced by glaucoma surgery with or without EX-PRESS and tube might have led to the formation of a thicker conjunctival bleb wall and ended in equivalent IOP values. Since aqueous humor is absorbed through the vessels of the conjunctival bleb wall, less inflammation might be desirable.

Other investigators have evaluated the biocompatibility of these devices using different methods. A modified semiquantitative grading system was used to assess cellularity and collagen deposition between EX-PRESS and tube groups in 1 study [4]. The mean number of goblet cells per high-power field from bleb sections of specimens were also calculated. Although they did not use untreated control eyes, they concluded that there was no difference in the capsule composition or inflammatory reaction between the 2 groups. Quantitative distribution of $\alpha$-SMA-positive cells, which represents the extent of tissue scarring, was evaluated, and again no significant differences were detected between trabeculectomy and EX-PRESS groups [13].

Our study provides information on the histocompatibility of conventional trabeculectomy versus the stainless-steel EX-PRESS or a silicone tube implant. However, there are some limitations to our study. First, this study included a relatively small sample size and a short observation period of 6 weeks. Second the study used a wellestablished rabbit filtration surgery model; however, the 
model is not a glaucoma model, and the result may not directly correlate with findings in humans. Third, some reports showed that the therapeutic effects of trabeculectomy and EX-PRESS are equivalent $[24,25]$. Actually, our study also showed that there were no differences between the EX-PRESS group and the trabeculectomy group in IOP, collagen area, bleb thickness, and inflammation area. However, in the EX-PRESS group, lower inflammation was reported compared with the trabeculectomy, which is contrary to our results [25]. These contradictions about inflammation might be due to the following points. Arimura et al. [25] demonstrated that inflammation was lower in the EX-PRESS group than the trabeculectomy group using a flare meter (FM-600, Kowa, Tokyo, Japan). On the other hand, the present study evaluated inflammation using Masson's trichrome staining. This difference might also cause the contradictions.

In conclusion, considering the similar outcomes noted after glaucoma filtration surgery with either the EXPRESS or silicone tube in our study, both implants appear to be relatively inert, with little difference in biocompatibility and bleb survival. However, since some degree of inflammatory bleb reaction was still observed histologically, and a correlation between bleb wall thickness and inflammation was found, more noninvasive surgical methods and more biocompatible materials may be desirable for less inflammation.

\section{Statement of Ethics}

All experiments were performed in accordance with the Association for Research in Vision and Ophthalmology (ARVO) Statement for the Use of Animals in Ophthalmic and Vision Research, and were approved and monitored by the Institutional Animal Care and Use Committee of Gifu Pharmaceutical University.

\section{Conflict of Interest Statement}

All authors have no commercial interests in any of the materials discussed in this article.

\section{Funding Sources}

This study was supported in part by the Japanese Society for the Promotion of Science (JSPS; Kakenhi grant number No. JP 26462634).

\section{Author Contributions}

All authors take responsibility for the integrity of the data and the accuracy of the data analysis. K.I.: study concept and design. K.I., Y.N., K.O., and T.O.: contribution to scientific experiments. K.I. and S.I.: drafting of the manuscript. M.S., K.K., H.H., and T.Y.: critical revision of the manuscript for important intellectual content.

\section{References}

1 Shaarawy T, Goldberg I, Fechtner R. EXPRESS glaucoma filtration device: review of clinical experience and comparison with trabeculectomy. Surv Ophthalmol. 2015 JulAug;60(4):327-45.

2 Coleman AL. Advances in glaucoma treatment and management: surgery. Invest Ophthalmol Vis Sci. 2012 May;53(5):2491-4.

3 Xi L, Wang T, Zhao F, Zheng Q, Li X, Luo J, et al. Evaluation of an injectable thermosensitive hydrogel as drug delivery implant for ocular glaucoma surgery. PLoS One. 2014 Jun; 9(6):e100632.

4 Laing AE, Seibold LK, SooHoo JR, Kahook MY. Evaluation of bleb characteristics after implantation of the EX-PRESS ${ }^{\mathrm{TM}}$ glaucoma filtration device. Mol Vis. 2012;18:10-3.

5 Wang W, Zhang X. Meta-analysis of randomized controlled trials comparing EX-PRESS implantation with trabeculectomy for openangle glaucoma. PLoS One. 2014 Jun; 9(6):e100578.

6 Wang L, Sha F, Guo DD, Bi HS, Si JK, Du YX, et al. Efficacy and economic analysis of ExPRESS implantation versus trabeculectomy in uncontrolled glaucoma: a systematic review and meta-analysis. Int $\mathrm{J}$ Ophthalmol. 2016 Jan;9(1):124-31.

7 Chan JE, Netland PA. EX-PRESS Glaucoma Filtration Device: efficacy, safety, and predictability. Med Devices (Auckl). 2015 Sep;8: 381-8.

8 Ishida K, Moroto N, Murata K, Yamamoto T. Effect of glaucoma implant surgery on intraocular pressure reduction, flare count, anterior chamber depth, and corneal endothelium in primary open-angle glaucoma. Jpn J Ophthalmol. 2017 Jul;61(4):334-46.

9 Nyska A, Glovinsky Y, Belkin M, Epstein Y. Biocompatibility of the Ex-PRESS miniature glaucoma drainage implant. J Glaucoma. 2003 Jun;12(3):275-80.

10 De Feo F, Jacobson S, Nyska A, Pagani P, Traverso CE. Histological biocompatibility of a stainless steel miniature glaucoma drainage device in humans: a case report. Toxicol Pathol. 2009 Jun;37(4):512-6.

11 Aziz H, Fantes F, Dubovy S. Histopathology of the Ex-PRESS Shunt. Ophthalmic Surg Lasers Imaging. 2011;42 Online:e94-6.
12 Konstantinidis A, Panos GD, Triantafylla M, Labiris G, Tsaragli E, Gatzioufas Z, et al. Imaging of filtering blebs after implantation of the Ex-PRESS shunt with the use of the Visante optical coherence tomography. Int J Ophthalmol. 2015 Jun;8(3):492-5.

13 Shobayashi K, Inoue T, Kawai M, Iwao K, Ohira S, Kojima S, et al. Postoperative Changes in Aqueous Monocyte Chemotactic Protein-1 Levels and Bleb Morphology after Trabeculectomy vs. Ex-PRESS Shunt Surgery. PLoS One. 2015 Oct;10(10): e0139751.

14 Schaefer JL, Levine MA, Martorana G, Koenigsman H, Smith MF, Sherwood MB. Failed glaucoma drainage implant: long-term outcomes of a second glaucoma drainage device versus cyclophotocoagulation. Br J Ophthalmol. 2015 Dec;99(12):1718-24.

15 Lee NY, Hwang HB, Oh SH, Park CK. Efficacy of Additional Glaucoma Drainage Device Insertion in Refractory Glaucoma: Case Series with a Systematic Literature Review and Meta-Analysis. Semin Ophthalmol. 2015; 30(5-6):345-51. 
16 Lloyd MA, Baerveldt G, Nguyen QH, Minckler DS. Long-term histologic studies of the Baerveldt implant in a rabbit model. J Glaucoma. 1996 Oct;5(5):334-9.

17 Ayyala RS, Harman LE, Michelini-Norris B, Ondrovic LE, Haller E, Margo CE, et al. Comparison of different biomaterials for glaucoma drainage devices. Arch Ophthalmol. 1999 Feb;117(2):233-6.

18 Ayyala RS, Michelini-Norris B, Flores A, Haller E, Margo CE. Comparison of different biomaterials for glaucoma drainage devices: part 2. Arch Ophthalmol. 2000 Aug;118(8): 1081-4.

19 Ishida K, Netland PA, Costa VP, Shiroma L, Khan B, Ahmed II. Comparison of polypropylene and silicone Ahmed glaucoma valves. Ophthalmology. 2006 Aug;113(8):1320-6.
20 Jung KI, Lee SB, Kim JH, Park CK. Foreign body reaction in glaucoma drainage implant surgery. Invest Ophthalmol Vis Sci. 2013 Jun; 54(6):3957-64.

21 Good TJ, Kahook MY. Assessment of bleb morphologic features and postoperative outcomes after Ex-PRESS drainage device implantation versus trabeculectomy. Am J Ophthalmol. 2011 Mar;151(3):507-13.e1.

22 Joseph JP, Grierson I, Hitchings RA. Chemotactic activity of aqueous humor. A cause of failure of trabeculectomies? Arch Ophthalmol. 1989 Jan;107(1):69-74.
23 Granstein RD, Staszewski R, Knisely TL, Zeira E, Nazareno R, Latina $M$, et al. Aqueous humor contains transforming growth factorbeta and a small (less than 3500 daltons) inhibitor of thymocyte proliferation. J Immunol. 1990 Apr;144(8):3021-7.

24 Wang W, Zhou MW, Huang WB, Gao XB, Zhang XL. Ex-PRESS implantation versus trabeculectomy in Chinese patients with POAG: fellow eye pilot study. Int J Ophthalmol. 2017 Jan;10(1):56-60.

25 Arimura S, Takihara Y, Miyake S, Iwasaki K, Gozawa M, Matsumura T, et al. Randomized Clinical Trial for Early Postoperative Complications of Ex-PRESS Implantation versus Trabeculectomy: Complications Postoperatively of Ex-PRESS versus Trabeculectomy Study (CPETS). Sci Rep. 2016 May;6(1): 26080. 